\title{
Stereotactic body radiation therapy versus radiofrequency ablation for single small hepatocellular carcinoma: a propensity-score matching analysis of their impact on liver function and clinical outcomes
}

\author{
Masayuki Ueno ${ }^{1,2}$, Hiroyuki Takabatake ${ }^{1}$, Satoshi Itasaka ${ }^{3}$, Takahisa Kayahara ${ }^{1}$, Youichi Morimoto ${ }^{1}$, \\ Hiroshi Yamamoto ${ }^{1}$, Motowo Mizuno ${ }^{1}$ \\ ${ }^{1}$ Department of Gastroenterology and Hepatology, Kurashiki Central Hospital, Okayama, Japan; ${ }^{2}$ Department of Gastroenterology and Hepatology, \\ Graduate School of Medicine, Kyoto University, Kyoto, Japan; ${ }^{3}$ Department of Radiation Oncology, Kurashiki Central Hospital, Okayama, Japan \\ Contributions: (I) Conception and design: M Ueno, H Takabatake, T Kayahara, Y Morimoto; (II) Administrative support: None; (III) Provision \\ of study materials or patients: None; (IV) Collection and assembly of data: M Ueno, S Itasaka; (V) Data analysis and interpretation: M Ueno, H \\ Takabatake, T Kayahara, Y Morimoto, H Yamamoto, M Mizuno; (VI) Manuscript writing: All authors; (VII) Final approval of manuscript: All \\ authors. \\ Correspondence to: Masayuki Ueno. Department of Gastroenterology and Hepatology, Kurashiki Central Hospital, 1-1-1 Miwa, Kurashiki, Okayama \\ 710-8602, Japan. Email: mu13951@kchnet.or.jp.
}

\begin{abstract}
Background: Stereotactic body radiation therapy (SBRT) has high efficacy for early-stage hepatocellular carcinoma (HCC) and is an accepted alternative to radiofrequency ablation (RFA). However, SBRT for HCC may cause subacute liver injury leading to negative clinical outcomes. In this study, we compared changes of liver function and prognosis after SBRT or RFA in patients with single, small HCC by using a propensityscore matching analysis.
\end{abstract}

Methods: We reviewed medical records of 140 patients with single $\leq 3 \mathrm{~cm}$ HCC treated with SBRT or RFA at Kurashiki Central Hospital between January 2014 and February 2019. Changes of albumin-bilirubin (ALBI) score, local recurrence, and overall survival were compared between the propensity-score matched groups (31 patients treated with SBRT and 62 treated with RFA).

Results: The ALBI score increased modestly but significantly after SBRT, while it was unchanged in the RFA group; the intergroup difference was statistically significant $(\mathrm{P}=0.004)$. No local recurrence was identified in the SBRT group, whereas the cumulative recurrence incidence was $9.7 \%$ in the RFA group $(\mathrm{P}=0.023)$. Overall survival was not significantly different between the two groups (hazard ratio: $1.32,95 \%$ confidence interval: $0.60-2.89, \mathrm{P}=0.401)$.

Conclusions: SBRT had modestly negative impact on liver function but with appraisable local control of HCC. Our findings should contribute to the selection of this modality for treatment of single, small HCC.

Keywords: Hepatocellular carcinoma (HCC); stereotactic body radiation therapy (SBRT); radiofrequency ablation (RFA); liver function; propensity-scores

Submitted Jun 18, 2021. Accepted for publication Sep 06, 2021.

doi: 10.21037/jgo-21-356

View this article at: https://dx.doi.org/10.21037/jgo-21-356

\footnotetext{
$\wedge$ ORCID: 0000-0002-2497-9327.
} 


\section{Introduction}

Hepatocellular carcinoma (HCC) is the fourth most common cause of cancer-related death worldwide (1). Curative treatment at an early stage is a cornerstone for improving the outcome of HCC (2). According to current guidelines, surgical resection, radiofrequency ablation (RFA), or liver transplantation is recommended for treatment of early-stage HCC (3). However, each treatment has drawbacks: surgical resection is invasive and may cause postoperative morbidity and liver failure (4); the therapeutic efficacy with RFA may be limited by tumor size and its location $(5,6)$; and, because of the scarcity of donor organs, liver transplantation is rarely performed in Asian countries (7).

Remarkable technological advances in radiotherapy for HCC have been made in recent years (8). Stereotactic body radiation therapy (SBRT) uses hypo-fractionated irradiation with a very high dose per fraction, which enables satisfactory therapeutic effects with lower toxicity (8). The local control rate of SBRT for early-stage HCC has been reported to be $87-100 \%(9-11)$. SBRT is expected to overcome the drawbacks of surgical resection and RFA because it is less invasive than resection, and it is less influenced by tumor size and location than is RFA (6).

However, important issues with SBRT remain to be clarified. First, SBRT can cause radiation-induced liver disease (RILD) $(12,13)$. Thanks to modern radiotherapy techniques, classic RILD (i.e., hepatomegaly, ascites, and elevated liver enzymes) is rare in the current era (14). Nonetheless, non-classic RILD, which also is associated with worse prognosis, sometimes occurs (13), and the longterm impact of SBRT on liver function is uncertain. Second, survival outcomes with SBRT are variable; a multi-center study reported better overall survival with SBRT than with RFA $(6,15)$, whereas an analysis using the National Cancer Database reported an opposite result $(16,17)$. In this study, using a propensity-score matching analysis, we compared the long-term impact on liver function and clinical outcomes of patients with single, small HCC treated with SBRT or RFA. We present the following article in accordance with the STROBE reporting checklist (available at https://dx.doi.org/10.21037/jgo-21-356).

\section{Methods}

\section{Patients}

We conducted a retrospective study by reviewing medical records of 641 patients with HCC treated at Kurashiki
Central Hospital between January 2014 and February 2019. The inclusion criteria for this study were (I) a single HCC of $3 \mathrm{~cm}$ or smaller; (II) diagnosis established by histology or contrast-enhanced imaging, according to the Liver Imaging Reporting and Data System (18); (III) treatment with SBRT or RFA; and (IV) patients age 20 years old or older. The exclusion criteria were (I) macrovascular invasion or extrahepatic metastasis; (II) Child-Pugh score 10 or higher; and (III) refusal to join in the study. One hundred and forty patients were included in this study; 44 were treated with SBRT and 96 with RFA. In our institution, RFA was the first-choice therapy for single, small HCC, and SBRT was chosen when RFA was considered inappropriate (e.g., local recurrence after RFA and tumors adjacent to the vessels or liver surface). All procedures were performed in accordance with the Helsinki Declaration (as revised in 2013). This study was approved by the Medical Ethics Committee of Kurashiki Central Hospital (No. 2948). Informed consent was obtained from all patients included in the study in an opt-out protocol.

\section{SBRT procedure}

Patients were immobilized with use of an individually shaped vacuum pillow and underwent a four-dimensional (4D) computed tomography (CT) scan. The gross tumor volume was delineated with the assistance of contrastenhanced CT scans and magnetic resonance imaging (MRI). The 4D CT was used to help create an internal target volume to compensate for respiratory motion. The planning target volume (PTV) included the internal target volume plus a 3- to 5-mm expansion. A stereotactic, multiarc, dynamic, conformal radiation procedure or volumetric modulated arc therapy was performed by use of TrueBeam or Clinac IX (Varian Medical Systems, Palo Alto, CA, USA), with respiratory gating or breath hold. Target alignment and setup accuracy were ensured through use of implanted fiducial markers [Gold Anchor ${ }^{\mathrm{TM}}$ (Naslund Medical AB, Huddinge, Sweden) or VISICOIL ${ }^{\mathrm{TM}}$ (IZI Medical Products, Baltimore, MD, USA)]. A total dose of 40 Gy was delivered in five fractions over 5-9 days on either an inpatient or outpatient basis.

\section{RFA procedure}

RFA was performed with real-time ultrasonographic guidance on an inpatient basis. We used Cool-tip ${ }^{\mathrm{TM}} \mathrm{RF}$ Ablation System E Series (Covidien, Mansfield, TX, 
USA) and tried to create an ablative margin of at least $5 \mathrm{~mm}$ surrounding the tumor; the margin setting was modified appropriately based on tumor location and its aggressiveness. Switching RFA with multiple electrodes was not conducted. Contrast-enhanced CT or nonenhanced MRI was performed on the day after RFA; if ablation was incomplete, an additional session was conducted during the same hospital stay.

\section{Follow-up}

After treatment, patients were assessed every 3 months with laboratory and radiological examination. Contrast-enhanced CT or MRI was performed at least every 6 months unless patients had contraindications to contrast administration. Post-treatment response was assessed according to the Response Evaluation Criteria in Cancer of the Liver (19). If HCC recurred, appropriate treatment was provided based on the clinical guideline (3).

\section{Statistical analysis}

Categorical variables were expressed as numbers (percentages) and continuous variables were expressed as median [interquartile range] or mean \pm standard deviation. To compare differences in patients' characteristics between the two groups, Fisher's exact test and the Mann-Whitney U test were used for categorical values and continuous values, respectively. Longitudinal changes of albumin-bilirubin (ALBI) score, local recurrence, and overall survival were compared between the propensity-score matched groups. Patients in the SBRT group were matched at a 1:2 ratio with patients treated with RFA. Propensity-score matching was performed without replacement and with a caliper of 0.2 . The variables included in the propensity-score model were age, tumor size, performance status, baseline ALBI score, baseline Child-Pugh score, and the number of previous treatments for HCC. To achieve good matching of baseline liver function, minor characteristics such as sex and etiology were not included in the propensity-score model. Tumor location was excluded from the model because the intergroup difference was too large to be matched. Barcelona Clinic Liver Cancer (BCLC) staging was also not included because the stage was determined by other variables (tumor size, performance status, and Child-Pugh score).

Time to local recurrence was defined as the time from SBRT or RFA to radiologic diagnosis of local recurrence.
Overall survival was defined as the time from SBRT or RFA to all-cause death, with living patients censored on the date of last follow-up. In each treatment group, ALBI scores at 12 months after treatment were compared with the baseline values by using the Wilcoxon rank sum test. To analyze the intergroup difference of the longitudinal change of ALBI scores, repeated measures analysis of variance was employed. Some values were missing because of death or loss to follow-up; thus, we used complete-case analysis. In the SBRT group, the relationship between PTV and absolute difference of ALBI scores (baseline vs. 12 months after treatment) was studied by using Pearson's product-moment correlation coefficient. Kaplan-Meier analysis, the log-rank test, and the Cox proportional hazards models were used for analysis of local recurrence and overall survival. A two-tailed $\mathrm{P}$ value of $<0.05$ was considered statistically significant. All statistical analyses were performed with R (version 3.5.1, R Foundation) and JMP (version 12.2, SAS Institute, Japan).

\section{Results}

\section{Baseline characteristics}

Among the 140 patients, 101 (72.1\%) were male, and the median age was 75 [67.3-81] years. Most patients (80.7\%) had well-compensated liver function (Child-Pugh class A). Median tumor size was 13 [10-20] mm. Before propensityscore matching (Table 1), patients in the SBRT group had significantly higher age, worse performance status, higher proportion of stage A tumors, and more patients having had previous treatment than did those in the RFA group. In addition, tumor location was significantly different and SBRT was more frequently performed as salvage therapy for recurrence after RFA or transcatheter arterial chemoembolization (TACE). SBRT was implemented in several circumstances: when tumors were adjacent to vessels $(\mathrm{n}=25)$, adjacent to the liver surface or the diaphragm $(\mathrm{n}=6)$, adjacent to the biliary tracts $(n=3)$, or near the heart $(n=3)$; local recurrence after RFA ( $\mathrm{n}=3$ ); tumor undetectable with ultrasonography $(n=1)$; no safe ablation route $(n=1)$; the presence of a pacemaker $(n=1)$; and patient's choice $(n=1)$. After the matching (Table 2, Table S1), 31 patients treated with SBRT were compared with 62 patients treated with RFA. ALBI scores and other baseline characteristics were well balanced between the two groups, but patients with hepatitis $\mathrm{C}$ virus $(\mathrm{HCV})$ infection were more numerous in the RFA group (41/62, $66.1 \%$ vs. $13 / 62,41.9 \% ; \mathrm{P}=0.044)$, and the frequency of salvage therapy was significantly 
Table 1 Patients' baseline characteristics before propensity-score matching

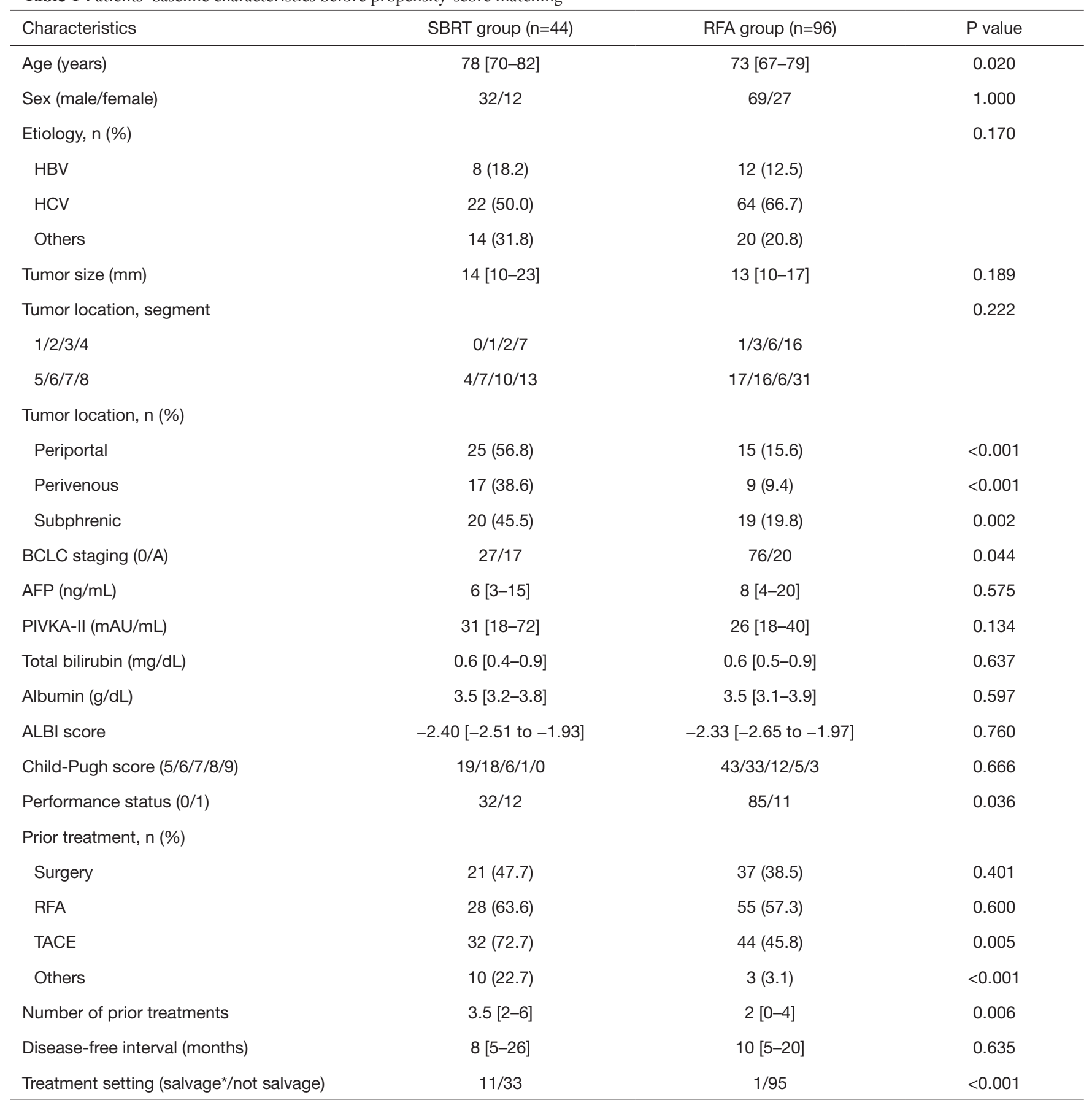

*, recurrence after RFA or TACE. SBRT, stereotactic body radiation therapy; RFA, radiofrequency ablation; HBV, hepatitis $B$ virus; HCV, hepatitis C virus; BCLC, Barcelona Clinic Liver Cancer; AFP, alpha-fetoprotein; PIVKA, protein induced by vitamin K absence or antagonists; ALBI, albumin-bilirubin; TACE, transcatheter arterial chemoembolization.

higher in the SBRT group $(8 / 23,25.8 \%$ vs. $1 / 16,1.6 \%$, $\mathrm{P}<0.001)$. Perivascular and subphrenic tumors were more common in the SBRT group. In HCV-infected patients, antiviral therapy would have influenced the longitudinal change of liver function, but only a small number of patients received antiviral therapy within 1 year after SBRT or RFA 
Table 2 Patients' baseline characteristics after propensity-score matching

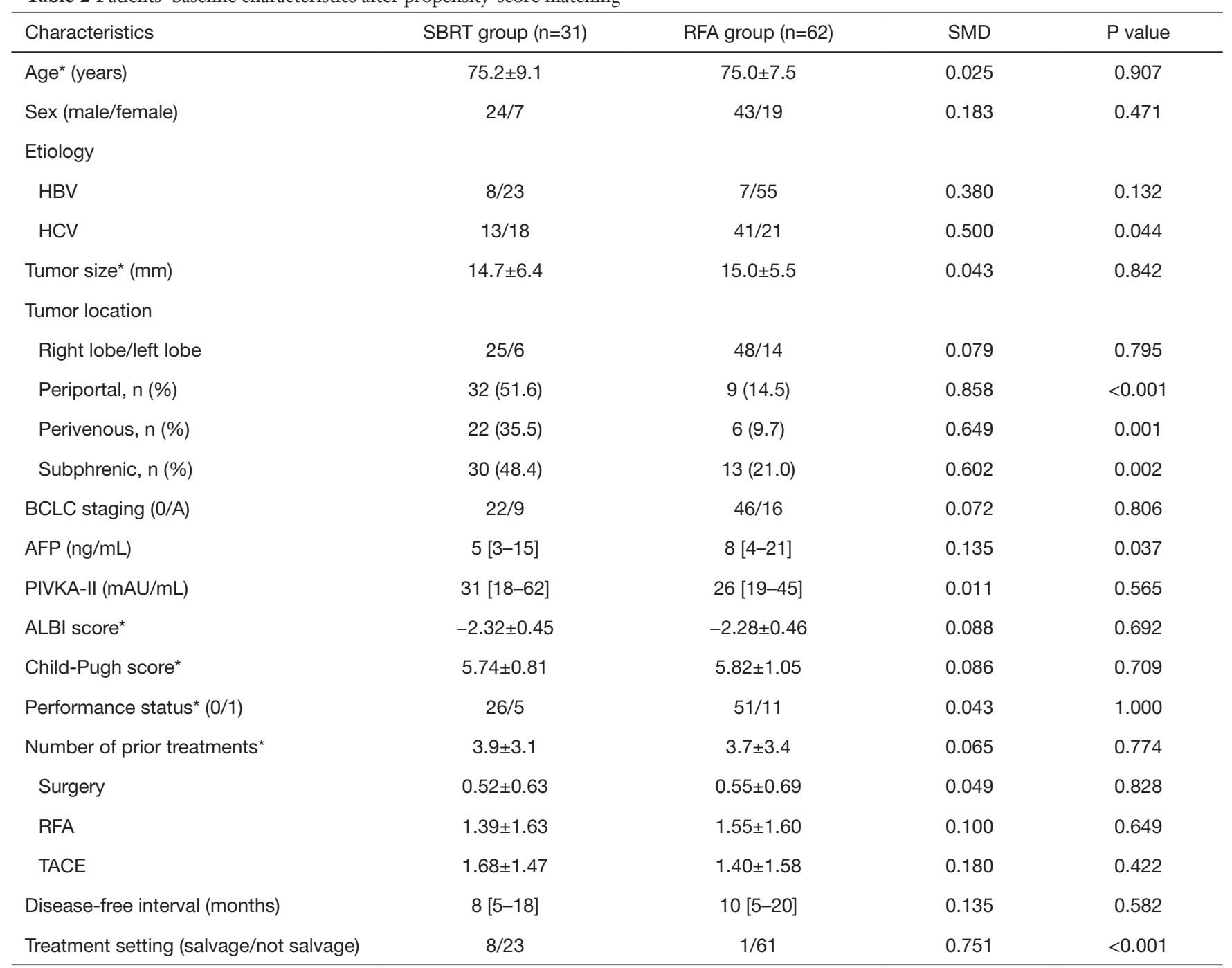

*, variables included in the propensity score model. SBRT, stereotactic body radiation therapy; RFA, radiofrequency ablation; SMD, standardized mean difference; HBV, hepatitis B virus; HCV, hepatitis C virus; BCLC, Barcelona Clinic Liver Cancer; AFP, alpha-fetoprotein; PIVKA, protein induced by vitamin $\mathrm{K}$ absence or antagonists; ALBI, albumin-bilirubin; TACE, transcatheter arterial chemoembolization.

treatment (Table S2).

\section{Longitudinal changes of ALBI score}

Figure 1 illustrates the longitudinal ALBI scores in the two treatment groups. In the SBRT group, the ALBI score gradually increased (i.e., liver function got worse) between the baseline and 12 months after treatment $(-2.32 \pm 0.45 v s$. $-2.17 \pm 0.61, \mathrm{P}<0.001)$ (Figure $1 A)$. In contrast, the ALBI score was unchanged in the RFA group $(-2.28 \pm 0.46$ at the baseline vs. $-2.30 \pm 0.55$ at 12 months after treatment, $\mathrm{P}=0.839$ ) (Figure 1B). Table 3 presents the numerical values for the longitudinal changes in ALBI values for the SBRT and RFA groups; the intergroup difference of the longitudinal changes of ALBI scores was statistically significant in the propensity-score matched cohort $(\mathrm{P}=0.004)$ and in the whole cohort $(\mathrm{P}=0.034)$. No significant difference was observed when we excluded the patients with diseasefree interval of less than 24 months $(\mathrm{P}=0.531)$. Figure 2 illustrates the subgroup analysis stratified by baseline modified ALBI (mALBI) grade (20); whereas ALBI scores increased after SBRT in patients who had reduced liver function (mALBI grade 2a, $\mathrm{P}=0.007$; and $2 \mathrm{~b}, \mathrm{P}=0.041$ ) at baseline, scores did not increase in the patients who had 
A

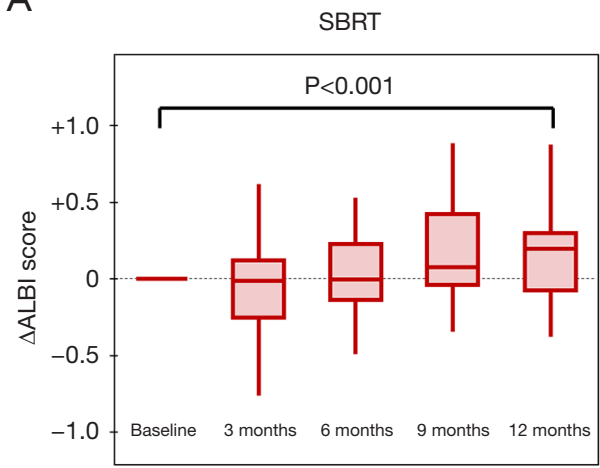

B

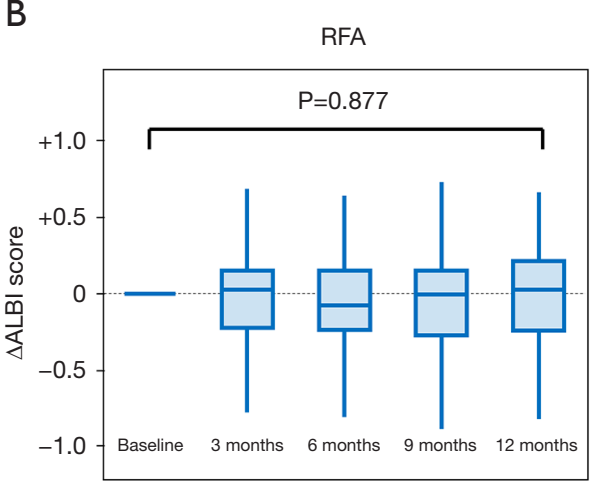

Figure 1 Longitudinal changes of ALBI score in (A) SBRT group and (B) RFA group. Differences of ALBI scores from baseline ( $\triangle$ ALBI scores) are shown in the figure. Vertical lines represent the ranges and box contains values between 25 th and 75 th percentiles (central line, median). ALBI scores at 12 months in the SBRT group were significantly increased from baseline, whereas scores were unchanged in the RFA group. ALBI, albumin-bilirubin; SBRT, stereotactic body radiation therapy; RFA, radiofrequency ablation.

Table 3 Longitudinal change of ALBI score after treatment

\begin{tabular}{|c|c|c|c|}
\hline Types of cohorts & SBRT group & RFA group & $\mathrm{P}_{\text {interaction }}{ }^{*}$ \\
\hline Baseline & $-2.32 \pm 0.45$ & $-2.28 \pm 0.46$ & \\
\hline 3 months & $-2.33 \pm 0.48$ & $-2.29 \pm 0.43$ & \\
\hline 6 months & $-2.28 \pm 0.47$ & $-2.31 \pm 0.57$ & \\
\hline 12 months & $-2.17 \pm 0.61$ & $-2.30 \pm 0.55$ & \\
\hline Cohort before the matching $(n=140)$ & & & 0.034 \\
\hline Baseline & $-2.26 \pm 0.52$ & $-2.29 \pm 0.49$ & \\
\hline 3 months & $-2.28 \pm 0.55$ & $-2.30 \pm 0.47$ & \\
\hline 12 months & $-2.16 \pm 0.68$ & $-2.30 \pm 0.56$ & \\
\hline \multicolumn{3}{|c|}{ Cohort excluding patients with disease-free interval of $<24$ months $(n=57)$} & 0.531 \\
\hline Baseline & $-2.35 \pm 0.61$ & $-2.41 \pm 0.48$ & \\
\hline 3 months & $-2.44 \pm 0.68$ & $-2.43 \pm 0.47$ & \\
\hline 6 months & $-2.42 \pm 0.68$ & $-2.49 \pm 0.55$ & \\
\hline 9 months & $-2.33 \pm 0.76$ & $-2.59 \pm 0.45$ & \\
\hline 12 months & $-2.39 \pm 0.69$ & $-2.52 \pm 0.44$ & \\
\hline
\end{tabular}

*, repeated measures analysis of variance. ALBI, albumin-bilirubin; SBRT, stereotactic body radiation therapy; RFA, radiofrequency ablation. 


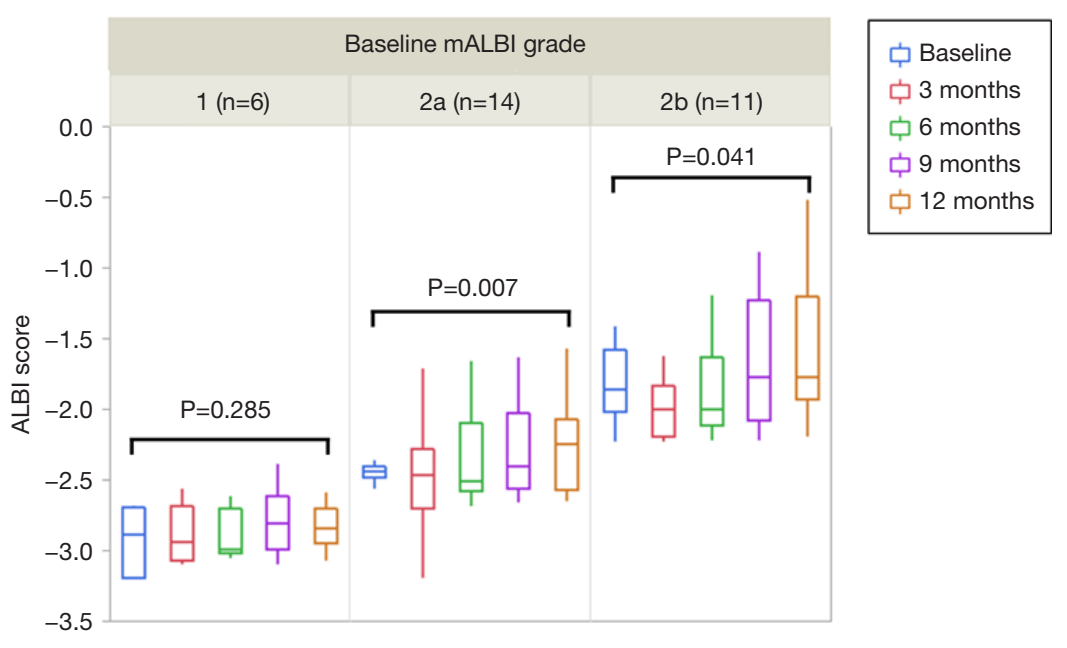

Figure 2 Subgroup analysis of the longitudinal changes of ALBI score after SBRT. The whole cohort was divided into three subgroups according to the baseline mALBI grade. ALBI, albumin-bilirubin; SBRT, stereotactic body radiation therapy; mALBI, modified ALBI.
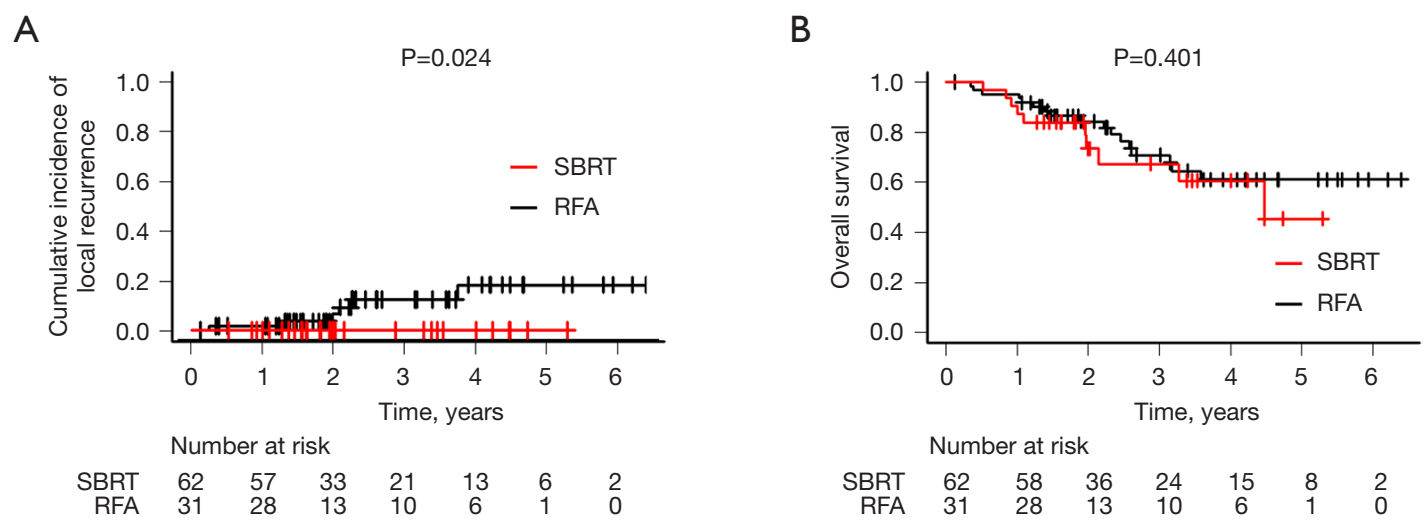

Figure 3 Kaplan-Meier analysis of cumulative incidence of local recurrence (A) and overall survival (B). SBRT, stereotactic body radiation therapy; RFA, radiofrequency ablation.

good ALBI score (mALBI grade $1, \mathrm{P}=0.285$ ) at baseline. During the 12 months after therapy, intrahepatic recurrence of HCC including local recurrence was identified in 9 patients in the SBRT group (29.0\%) and 19 patients in the RFA group (30.6\%), a statistically insignificant difference $(\mathrm{P}=1.000)$. This result is evident that recurrence of HCC was not responsible for differences in longitudinal changes of ALBI scores in the two groups. In the SBRT group, medians of gross tumor volume and PTV were 3.4 (range, $0.5-32.0) \mathrm{mm}^{3}$ and 27 (range, 5-104) $\mathrm{mm}^{3}$, respectively. PTV was not significantly associated with absolute difference of ALBI scores (baseline vs. 12 months after SBRT); the correlation coefficient $=-0.22$ and $\mathrm{P}=0.191$ ) (Figure S1). Besides worsening of liver function, no specific treatment-related toxicity was detected, except one case with acute cholecystitis after SBRT.

\section{Local recurrence and overall survival}

Figure $3 A$ illustrates that during the follow-up periods of up to 63.3 (median, 23.4) months in the SBRT group and up to 76.6 (median, 27.1) months in the RFA group, no local recurrence of HCC was identified in the SBRT group, whereas 6 cases were identified in the RFA group, a cumulative incidence of $9.7 \%(\mathrm{P}=0.024)$. Figure $3 B$ illustrates that during the follow-up periods, $10(32.3 \%)$ patients in the SBRT group and $17(27.4 \%)$ patients in the RFA group died, a statistically insignificant difference 
(hazard ratio: $1.32,95 \%$ confidence interval: $0.60-2.89$, $\mathrm{P}=0.401$ ). The cumulative incidence of all-cause mortality at 5 years was $32.3 \%$ in the SBRT group and $43.5 \%$ in the RFA group. When we excluded patients with disease-free intervals less than 24 months instead of propensity-score matching, no significant intergroup difference was observed in the local recurrence $(6.3 \%$ in the SBRT group and $14.6 \%$ in the RFA group, $\mathrm{P}=0.660$ ) or overall survival (hazard ratio: $1.84,95 \%$ confidence interval: $0.33-10.35, \mathrm{P}=0.484$ ).

\section{Impact of increased ALBI score on survival outcomes}

In the SBRT group, ALBI score increased by 0.15 on average during 12 months follow-up. To investigate the clinical impact of the increase, we compared survival outcomes between patients with and without worsening of ALBI score. Increase of ALBI score (absolute difference $\geq 0.15$ ) was seen in 15 patients (48.4\%) in the SBRT group and 21 (33.9\%) patients in the RFA group. The increase of ALBI score was significantly associated with worse overall survival $(\mathrm{P}=0.024)$, whereas there was no significant association with localrecurrence free survival $(\mathrm{P}=0.628)$ (Figure S2).

\section{Discussion}

In this study of therapy for single, small HCC, SBRT had significantly worse impact on liver function, assessed by ALBI score, but achieved better local control than did RFA. Despite these differences, overall survival was similar between patients treated with the two procedures. To our best knowledge, this is the first study that compared, with propensity-score matching analysis, the long-term effects of SBRT and RFA on liver function.

In studies that have used Child-Pugh score for assessment of liver toxicity, the changes of liver function after treatment were not significantly different between SBRT- and RFA-treated patients $(15,21)$. We used ALBI score for assessment of liver function-which is reportedly a better predictor of the prognosis of HCC than Child-Pugh score (22) - to analyze the liver function as a continuous variable. Thus, we could analyze the delicate changes of liver function, which disclosed the differences between SBRT and RFA treatment.

The increases in ALBI score became noticeable more than 6 months after SBRT. This long interlude can be explained by the fact that, after SBRT, blood flow decreases and the necrotic parenchyma expands over several months $(23,24)$. We also found that increase of ALBI scores was associated with abnormal liver function (mALBI grade $2 \mathrm{a}$ and $2 \mathrm{~b}$ ) at baseline. This observation is in accordance with reports that worse liver function at baseline was associated with higher risk of RILD (25-27). Thus, careful follow-up of liver function should be continued for at least 1 year after SBRT, especially in patients with known impaired liver function.

Although liver toxicity, defined by increased ALBI score, was associated with SBRT more than with RFA, the degree was mild and not critical. Moreover, it should be appreciated that the excellent local control rate and the similar overall survival with SBRT was achieved despite the procedure being mostly performed for tumors that were difficult to completely ablate with RFA. Other studies also have found better local control of HCC with SBRT than with RFA, especially for perivascular tumors $(6,15,21)$. Since complete ablation is associated with better prognosis (28), the excellent local control with SBRT may also be beneficial. In contrast to our findings, an analysis of the National Cancer Database in the United State found SBRT inferior to RFA in survival benefit (14). However, that study did not include important variables, such as liver function, performance status, and treatment history for HCC, as matching factors. Other studies that balanced baseline liver function and previous treatment history found that survival outcomes with SBRT were similar to $(15,29)$ or even superior to outcomes with RFA (6). We also included the numbers of previous treatment and liver function in the propensity-score model and found that overall survival with SBRT was like that with RFA. Taken together, our findings suggest that SBRT is a promising treatment for early-stage HCC, especially for HCCs that are difficult to treat with RFA, despite its modest adverse effect on liver function.

The adverse effect on liver function in our patients treated with SBRT occurred despite our cautious use of that modality. Patients were treated with a total dose of $40 \mathrm{~Gy}$, TACE was not combined, and fiducial gold marker was used to compensate for respiratory liver motion. In other institutions, doses higher than 40 Gy together with TACE have been used $(30,31)$. The results of our subgroup analysis stratified by baseline liver function suggest that lower radiation dose (35 Gy) may be appropriate for patients with impaired liver function (15).

Limitations of our study are these: (I) it is a retrospective study, thus inherently flawed by selection and indication bias; though we performed propensity-score matching, unidentified confounding factors could have existed. (II) The sample size was relatively small; thus, some baseline characteristics including tumor location 
and treatment setting (salvage/not salvage) were not fully balanced even after matching. (III) The follow-up period was insufficient in some patients, which might have skewed the interpretation of survival outcomes. (IV) Most patients in this study had previous been treated for HCCs. When we excluded patients with disease-free intervals less than 24 months, no significant intergroup difference was observed in local recurrence or the longitudinal change of ALBI scores. Thus, our results cannot be simply applied to patients for treatment-naïve HCCs. To overcome these limitations, a prospective study (UMIN000036081) is ongoing in our institution to reassess the present results. (V) Dose and fractions of radiation used for HCC may not have been optimal. Although giving $40 \mathrm{~Gy}$ in five fractions is a commonly accepted protocol for treating $\operatorname{HCC}(15,31)$, the issue needs further study. (VI) Details of the RFA protocol differ among institutions or countries. Although we adopted the standard protocol in Japan (32), other procedures such as switching RFA using multiple electrode or CT-guided RFA are common in other countries. Switching RFA may provide better local tumor control than conventional RFA; however, the local recurrence rate in our study was not inferior to the results of previous studies with switching RFA (12.4-20.5\% at 36 months) $(33,34)$. Other studies have also shown that local control and survival outcomes did not differ between ultrasoundguided and CT-guided RFA (35,36).

\section{Conclusions}

SBRT had modestly negative impact on liver function but with appraisable local control of HCC in the real-world practice for single, small HCC. These advantage and disadvantage of SBRT should be considered in selecting the treatment modality, and long-term monitoring of liver function is advised after SBRT, especially in patients with pretreatment impaired liver function.

\section{Acknowledgments}

We are grateful to A. Takeda for helpful discussions and comments on the manuscript. We also thank W. R. Brown for the English language review.

Funding: None.

\section{Footnote}

Reporting Checklist: The authors have completed the
STROBE reporting checklist. Available at https://dx.doi. org/10.21037/jgo-21-356

Data Sharing Statement: Available at https://dx.doi. org/10.21037/jgo-21-356

Peer Review File: Available at https://dx.doi.org/10.21037/ jgo-21-356

Conflicts of Interest: All authors have completed ICMJE uniform disclosure form (available at https://dx.doi. org/10.21037/jgo-21-356). The authors have no conflicts of interest to declare.

Ethical Statement: The authors are accountable for all aspects of the work in ensuring that questions related to the accuracy or integrity of any part of the work are appropriately investigated and resolved. The study was conducted in accordance with the Declaration of Helsinki (as revised in 2013). The study was approved by the Medical Ethics Committee of Kurashiki Central Hospital (No. 2948). Informed consent was obtained from all patients included in the study in an opt-out protocol.

Open Access Statement: This is an Open Access article distributed in accordance with the Creative Commons Attribution-NonCommercial-NoDerivs 4.0 International License (CC BY-NC-ND 4.0), which permits the noncommercial replication and distribution of the article with the strict proviso that no changes or edits are made and the original work is properly cited (including links to both the formal publication through the relevant DOI and the license). See: https://creativecommons.org/licenses/by-nc-nd/4.0/.

\section{References}

1. Yang JD, Hainaut P, Gores GJ, et al. A global view of hepatocellular carcinoma: trends, risk, prevention and management. Nat Rev Gastroenterol Hepatol 2019;16:589-604.

2. Kudo M, Izumi N, Sakamoto $M$, et al. Survival analysis over 28 years of 173,378 patients with hepatocellular carcinoma in Japan. Liver Cancer 2016;5:190-7.

3. Marrero JA, Kulik LM, Sirlin CB, et al. Diagnosis, staging, and management of hepatocellular carcinoma: 2018 practice guidance by the American Association for the Study of Liver Diseases. Hepatology 2018;68:723-50.

4. Peng ZW, Lin XJ, Zhang YJ, et al. Radiofrequency 
ablation versus hepatic resection for the treatment of hepatocellular carcinomas $2 \mathrm{~cm}$ or smaller: a retrospective comparative study. Radiology 2012;262:1022-33.

5. Lee S, Kang TW, Cha DI, et al. Radiofrequency ablation vs. surgery for perivascular hepatocellular carcinoma: propensity score analyses of long-term outcomes. J Hepatol 2018;69:70-8.

6. Kim N, Cheng J, Jung I, et al. Stereotactic body radiation therapy vs. radiofrequency ablation in Asian patients with hepatocellular carcinoma. J Hepatol 2020;73:121-9.

7. Chan SC. Liver transplantation for hepatocellular carcinoma. Liver Cancer 2013;2:338-44.

8. Qiu B, Aili A, Xue L, et al. Advances in radiobiology of stereotactic ablative radiotherapy. Front Oncol 2020;10:1165.

9. Cárdenes HR, Price TR, Perkins SM, et al. Phase I feasibility trial of stereotactic body radiation therapy for primary hepatocellular carcinoma. Clin Transl Oncol 2010;12:218-25.

10. Bujold A, Massey CA, Kim JJ, et al. Sequential phase I and II trials of stereotactic body radiotherapy for locally advanced hepatocellular carcinoma. J Clin Oncol 2013;31:1631-9.

11. Kim JW, Seong J, Lee IJ, et al. Phase I dose escalation study of helical intensity-modulated radiotherapybased stereotactic body radiotherapy for hepatocellular carcinoma. Oncotarget 2016;7:40756-66.

12. Pan CC, Kavanagh BD, Dawson LA, et al. Radiationassociated liver injury. Int J Radiat Oncol Biol Phys 2010;76:S94-100.

13. Sanuki N, Takeda A, Oku Y, et al. Influence of liver toxicities on prognosis after stereotactic body radiation therapy for hepatocellular carcinoma. Hepatol Res 2015;45:540-7.

14. Koay EJ, Owen D, Das P. Radiation-induced liver disease and modern radiotherapy. Semin Radiat Oncol 2018;28:321-31.

15. Hara K, Takeda A, Tsurugai Y, et al. Radiotherapy for hepatocellular carcinoma results in comparable survival to radiofrequency ablation: a propensity score analysis. Hepatology 2019;69:2533-45.

16. Rajyaguru DJ, Borgert AJ, Smith AL, et al. Radiofrequency ablation versus stereotactic body radiotherapy for localized hepatocellular carcinoma in nonsurgically managed patients: analysis of the National Cancer Database. J Clin Oncol 2018;36:600-8.

17. Kudo M, Ikeda M, Ueshima K, et al. Response Evaluation Criteria in Cancer of the Liver version 5 (RECICL 2019 revised version). Hepatol Res 2019;49:981-9.

18. Chernyak V, Santillan CS, Papadatos D, et al. LIRADS® algorithm: CT and MRI. Abdom Radiol (NY) 2018;43:111-26.

19. Berger NG, Tanious MN, Hammad AY, et al. External radiation or ablation for solitary hepatocellular carcinoma: a survival analysis of the SEER database. J Surg Oncol 2017;116:307-12.

20. Hiraoka A, Michitaka K, Kumada T, et al. Validation and potential of albumin-bilirubin grade and prognostication in a nationwide survey of 46,681 hepatocellular carcinoma patients in Japan: the need for a more detailed evaluation of hepatic function. Liver Cancer 2017;6:325-36.

21. Kim N, Kim HJ, Won JY, et al. Retrospective analysis of stereotactic body radiation therapy efficacy over radiofrequency ablation for hepatocellular carcinoma. Radiother Oncol 2019;131:81-7.

22. Peng $\mathrm{Y}$, Wei Q, He Y, et al. ALBI versus child-pugh in predicting outcome of patients with HCC: a systematic review. Expert Rev Gastroenterol Hepatol 2020;14:383-400.

23. Price TR, Perkins SM, Sandrasegaran K, et al. Evaluation of response after stereotactic body radiotherapy for hepatocellular carcinoma. Cancer 2012;118:3191-8.

24. Mendiratta-Lala $M, \mathrm{Gu} E$, Owen D, et al. Imaging findings within the first 12 months of hepatocellular carcinoma treated with stereotactic body radiation therapy. Int J Radiat Oncol Biol Phys 2018;102:1063-9.

25. Jung J, Yoon SM, Kim SY, et al. Radiation-induced liver disease after stereotactic body radiotherapy for small hepatocellular carcinoma: clinical and dose-volumetric parameters. Radiat Oncol 2013;8:249.

26. Huang Y, Chen SW, Fan CC, et al. Clinical parameters for predicting radiation-induced liver disease after intrahepatic reirradiation for hepatocellular carcinoma. Radiat Oncol 2016;11:89.

27. Jun BG, Kim YD, Cheon GJ, et al. Clinical significance of radiation-induced liver disease after stereotactic body radiation therapy for hepatocellular carcinoma. Korean J Intern Med 2018;33:1093-102.

28. Sparchez Z, Mocan T, Radu P, et al. Prognostic factors after percutaneous radiofrequency ablation in the treatment of hepatocellular carcinoma. impact of incomplete ablation on recurrence and overall survival rates. J Gastrointestin Liver Dis 2018;27:399-407.

29. Wahl DR, Stenmark MH, Tao Y, et al. Outcomes after stereotactic body radiotherapy or radiofrequency ablation for hepatocellular carcinoma. J Clin Oncol 2016;34:452-9.

30. Baumann BC, Wei J, Plastaras JP, et al. Stereotactic body 
radiation therapy (SBRT) for hepatocellular carcinoma: high rates of local control with low toxicity. Am J Clin Oncol 2018;41:1118-24.

31. Kimura T, Takeda A, Sanuki N, et al. Multicenter prospective study of stereotactic body radiotherapy for previously untreated solitary primary hepatocellular carcinoma: the STRSPH study. Hepatol Res 2021;51:461-71.

32. Shiina S, Tateishi R, Arano T, et al. Radiofrequency ablation for hepatocellular carcinoma: 10-year outcome and prognostic factors. Am J Gastroenterol 2012;107:56977; quiz 578.

33. Choi JW, Lee JM, Lee DH, et al. Switching monopolar radiofrequency ablation using a separable cluster electrode in patients with hepatocellular carcinoma: a prospective

Cite this article as: Ueno $M$, Takabatake H, Itasaka S, Kayahara T, Morimoto Y, Yamamoto H, Mizuno M. Stereotactic body radiation therapy versus radiofrequency ablation for single small hepatocellular carcinoma: a propensityscore matching analysis of their impact on liver function and clinical outcomes. J Gastrointest Oncol 2021;12(5):2334-2344. doi: 10.21037/jgo-21-356 study. PLoS One 2016;11:e0161980.

34. Huang GL, Liu M, Zhang XE, et al. Multipleelectrode switching-based radiofrequency ablation vs. conventional radiofrequency ablation for single early-stage hepatocellular carcinoma ranging from 2 to $5 \mathrm{~cm}$. Front Oncol 2020;10:1150.

35. Lee LH, Hwang JI, Cheng YC, et al. Comparable outcomes of ultrasound versus computed tomography in the guidance of radiofrequency ablation for hepatocellular carcinoma. PLoS One 2017;12:e0169655.

36. Huo J, Aloia TA, Xu Y, et al. Comparative effectiveness of computed tomography- versus ultrasound-guided percutaneous radiofrequency ablation among medicare patients 65 years of age or older with hepatocellular carcinoma. Value Health 2019;22:284-92. 


\section{Supplementary}

Table S1 Details of previous treatments before SBRT and RFA after propensity-score matching

\begin{tabular}{lccc}
\hline Variables & SBRT group $(\mathrm{n}=31)$ & RFA group $(\mathrm{n}=62)$ & $\mathrm{P}$ value \\
\hline Any prior treatment (present/absent) & $28 / 3$ & $49 / 13$ & 0.247 \\
Surgery (present/absent) & $14 / 17$ & $27 / 35$ & 1.000 \\
Surgery, times & $0[0-1]$ & $0[0-1]$ & 0.956 \\
RFA (present/absent) & $19 / 12$ & $42 / 20$ & 0.644 \\
RFA, times & $1[0-3]$ & $1[0-2]$ & 0.505 \\
SBRT (present/absent) & $3 / 28$ & $4 / 58$ & 0.682 \\
SBRT, times & $0[0-0]$ & $0[0-0]$ & 0.630 \\
TACE (present/absent) & $22 / 9$ & $36 / 26$ & 0.262 \\
TACE, times & $2[0-3]$ & $1[0-2]$ & 0.291 \\
\hline
\end{tabular}

SBRT, stereotactic body radiation therapy; RFA, radiofrequency ablation; TACE, transcatheter arterial chemoembolization.

Table S2 Antiviral therapy for chronic hepatitis C infection

\begin{tabular}{lccc}
\hline Timing of treatment & SBRT group $(\mathrm{n}=13)$ & RFA group $(\mathrm{n}=41)$ & $\mathrm{P}$ value \\
\hline No treatment, $\mathrm{n}(\%)$ & $6(46.2)$ & $18(43.9)$ & 1.000 \\
Treated before SBRT/RFA, $\mathrm{n}(\%)$ & $6(46.2)$ & $6(14.6)$ & 0.170 \\
Treated within 1 year after SBRT/RFA, $\mathrm{n}(\%)$ & $1(7.7)$ & $7(17.1)$ & 1.000 \\
Treated more than 1 year after SBRT/RFA, $\mathrm{n}(\%)$ & $0(0.0)$ & 0.176 \\
\hline
\end{tabular}

SBRT, stereotactic body radiation therapy; RFA, radiofrequency ablation.

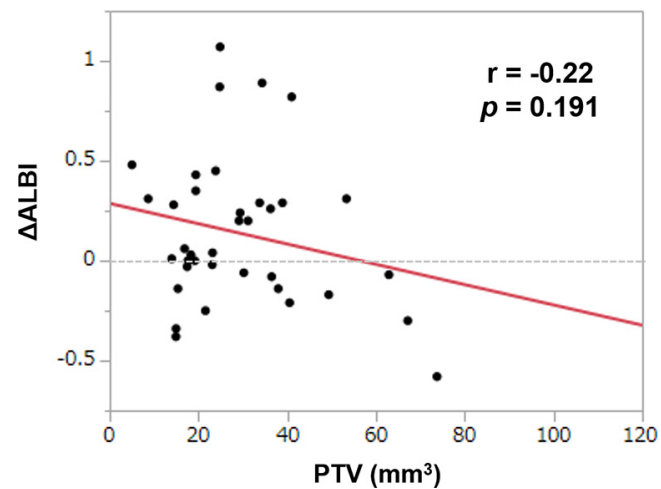

Figure S1 Correlation between PTV and absolute difference of ALBI scores ( $\triangle$ ALBI, baseline vs. 12 months after SBRT). PTV, planning target volume; ALBI, albumin-bilirubin; SBRT, stereotactic body radiation therapy. 
A Cumulative incidence of local recurrence

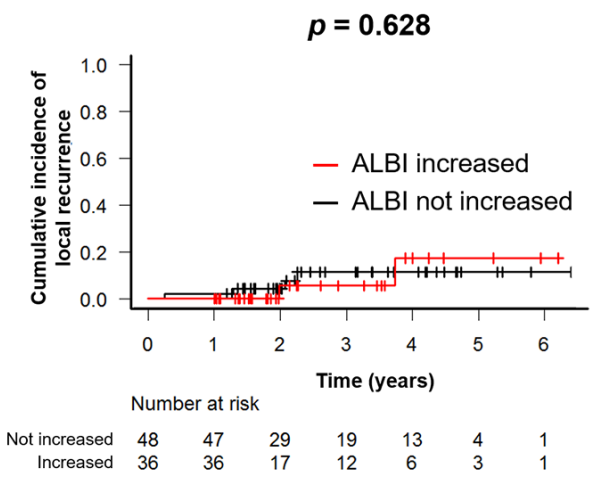

B

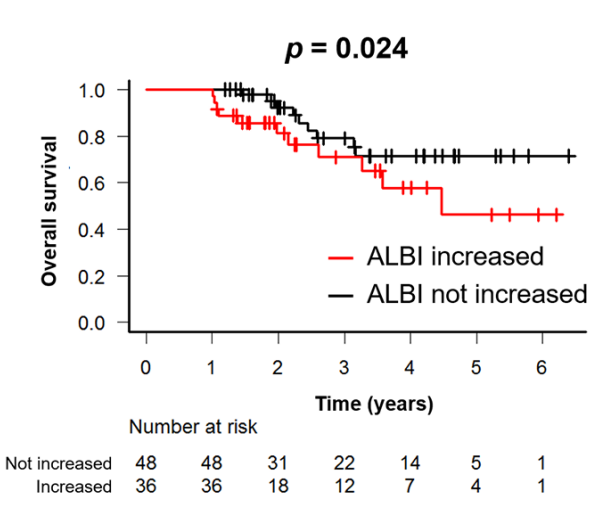

Figure S2 Comparison of cumulative incidence of local recurrence (A) and overall survival (B) between patients with and without ALBI score increase. ALBI, albumin-bilirubin. 